\title{
GNSS Based Passive Bistatic Radar for Micro-Doppler based Classification of Helicopters: Experimental Validation
}

\author{
C. Clemente, T. Parry, G. Galston, P. Hammond, C. Berry, C. Ilioudis, D. Gaglione and J. J. Soraghan \\ University of Strathclyde, CeSIP, EEE, 204 George Street, G1 1XW, Glasgow, UK \\ E-mail: carmine.clemente@ strath.ac.uk
}

\begin{abstract}
The capability of using illuminators of opportunity for target classification is of great interest to the radar community. In particular the alternative use of Global Navigation Satellite System (GNSS) has recently initiated a number of studies that aim to exploit this source of illumination for passive radar. We recently introduced the concept of a GNSS based passive radar for extraction of micro-Doppler signatures from helicopter rotor blades with the aim of identify these kind of targets. In this paper we present the experimental validation of our concept with real data from two different models of helicopter.

Index Terms-Passive Radar, Global Navigation Satellite System, micro-Doppler, forward scattering enhancement, experimental analysis.
\end{abstract}

\section{INTRODUCTION}

In recent years a big interest has been placed on the capability of using illuminators of opportunity for radar purposes. Possible illuminators are systems deployed generally for communication purposes, for example Digital Video BroadcastingTerrestrial (DVB-T), FM Radio, Wi-fi, Mobile Phone Networks, Global Navigation Satellite Systems (GNSS). A system exploiting the energy transmitted from these non-cooperative transmitters is called Passive Bistatic Radar and has been successfully investigated in different configurations and using different sources of illumination. This kind of technology has several advantages thus opening to many applications for civilian and military purposes. Exploiting an existing source of RF energy the system can be cheaper, stealthier, frequency allocation free and provide the capability to have information in portion of the spectrum unavailable in the past [1]. Recently this topic has seen the interest growing with an extensive work carried by the radar community. Real demonstrators [2], [3] have been built to prove the real applicability of this technology. A wide set of possible illuminators of opportunity have been evaluated [4], [5], [6], [7]. Moreover a set of more advanced radar techniques have been proved in this field such as through the wall radar [8], SAR and ISAR imaging [9], [10], reliable detection [11], [12] and Automatic Target Recognition [13].

We recently investigated the possibility to exploit the GNSS as an illuminator of opportunity to measure the micro-Doppler signature of helicopter rotor blades exploiting the favourable geometry and relatively low carrier frequency [14]. Micromotions present in a target, such as vibrations due to the engine or a rotating antenna introduces non stationary Doppler modulations named micro-Doppler [15]. These may be extracted from both monostatic and bistatic radar providing some interesting advantages in the bistatic case [15], [16], [17].The use of micro-Doppler for target classification has been deeply investigated in recent years [18], and it can be now considered a technology ready to be deployed in commercial devices [19]. Of particular interest in the paper is the analysis of the effect from the rotating rotor blades of an helicopter since the effect on the radar returns depends on the characteristics of the rotor like the blade rotating velocity, blade length and the number of blades [15], [20]. Helicopters parameters of helicopter rotor blades were estimated in [21] with an algorithm based on the Radon transform. This algorithm was tested on real data and shown to be accurate and able to recognize different helicopters, while in [22] the return of an helicopter in a DVB$\mathrm{T}$ based passive radar was analysed.

To deal with the problem of the extraction of the microDoppler signature of the rotor blades from the bulk return of the helicopter fuselage, clutter and direct signal interference in [23] we proposed the use of the Singular Spectrum Analysis (SSA) as tool to select the non stationary components belonging to the return of interest.

In this paper we report the experimental validation of the concept described in [14], providing an analysis of the returns from 2 different helicopters and showing the micro-Doppler signatures achieved after the SSA based extraction method [23]. The experimental results demonstrate the effectiveness of such a system for helicopter identification.

The remainder of paper is organized as follows. Section II summarizes the concept of the PBR presented in [14]. Section III describes the main receiver characteristics and the experiments, while Section IV presents the results showing the obtained micro-Doppler signatures from 2 helicopters. Section $\mathrm{V}$ concludes the paper.

\section{BISTATIC GNSS RADAR SYSTEM GEOMETRY AND SIGNAL MODEL}

The acquisition geometry of the passive bistatic radar proposed in [14] is shown in Figure 1. The transmitter is one of the GNSS transmitters visible from the receiver. $R_{T}$ and $R_{R}$ are the transmitter to target and the receiver to target ranges 
respectively. The angle $\beta$ is the solid bistatic angle defined as the solid angle between the transmitter, the target and the receiver [24].

The principal transmitted signal from the GNSS satellite

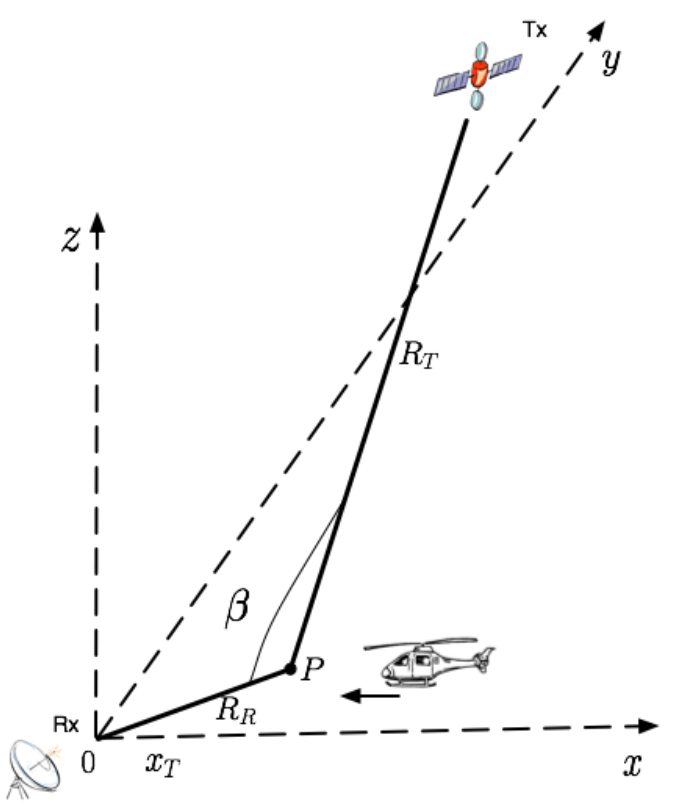

Figure 1: Proposed bistatic passive radar geometry with the GNSS illuminator.

is a Code Division Multiple Access code consisting of a Pseudo Random Noise (PRN) sequence, in particular for the GPS constellation that is exploited in this work, the sequence consists of a C/A code. The received radar return after the cross correlation with the replica of the PRN signal is [14]:

$$
s_{r c}(\tau, \eta)=A_{0} p_{r}\left(\tau-\frac{R_{R}(\eta)}{c}\right) \exp \left\{-j \frac{2 \pi f_{0} R(\eta)}{c}\right\}
$$

where $f_{0}$ is the carrier frequency, $c$ is the speed of light, $p_{r}(\tau)$ is the range envelope where the time reference is triggered to the direct signal received from the transmitter, $A_{0}$ is the amplitude of the scattered signal, $\tau$ is the variable representing the fast time of the received signal, while $\eta$ represents the slow time of the acquisition of the different echoed PRN sequences and $R(\eta)$ is the bistatic target range. This slow time is required because the dynamics of the micro-motion is slower than the dynamics of the signal used to perform ranging. This is also the reason why the cross-correlation of the C/A sequence can be performed without affecting the micro-Doppler analysis. However the signal model requires to be modified in the presence of target micro-motions where phase modulation of the signal is introduced.

In the application considered in this paper the receiver is a platform on ground. This kind of configuration allows us to obtain easily a near forward scattering configuration. The target is a helicopter approaching the Line Of Sight (LOS) between the transmitter and the receiver. It means that the bistatic angle $\beta$ is close to $180^{\circ}$ where forward

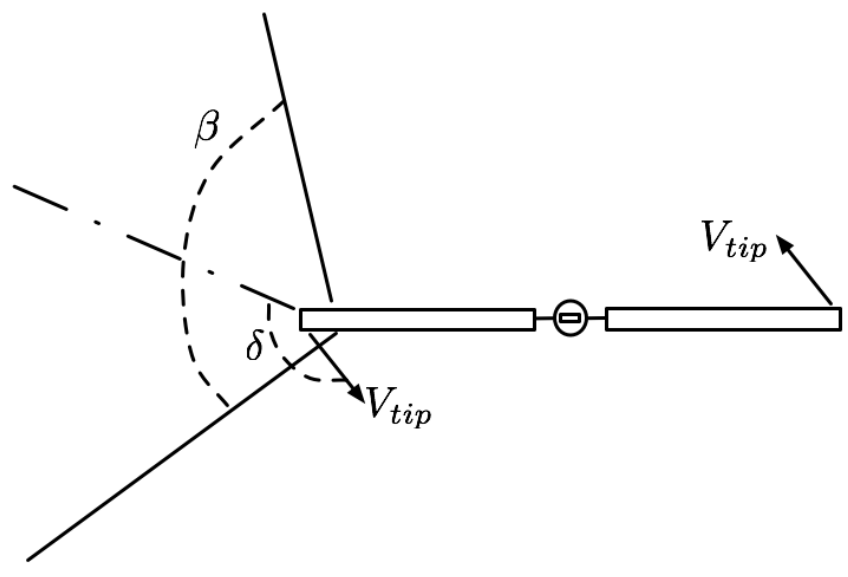

Figure 2: Geometry of the rotating blades seen from the bistatic radar.

scattering enhancement can be obtained [24]. This particular geometry is required in order to exploit the forward scattering enhancement increasing the radar cross section of the rotor blades. Further details about the power budget analysis can be found in [14].

Figure 2 shows a view of the geometry of the rotor blades relative to the bistatic geometry. The rotating plane of the rotor blade is parallel and orthogonal to the line of sight between the transmitter and the receiver. $\mathbf{V}_{\text {tip }}$ is the velocity vector of the tip of the rotor blade and $\delta$ is the angle between $\mathbf{V}_{\text {tip }}$ and the bisector of the bistatic angle $\beta$. The angles $\beta$ and $\delta$ are two important factors that influences the micro-Doppler signature of the target in a bistatic geometry [15].

The model of the received signal in (1) must consider also the effect of the micro-motion as follows:

$$
\begin{aligned}
s_{r c}(\tau, \eta)= & A_{1}(\eta) p_{r}\left(\tau-\frac{R_{R}(\eta)}{c}\right) \\
& \exp \left\{-j\left(\Phi_{R}(\eta)+\Phi_{m D}(\eta)\right)\right\}
\end{aligned}
$$

where $\Phi_{R}=2 \pi f_{0} R(\eta) / c$ is the phase of the target containing the constant motion and $\Phi_{m D}(\eta)$ is the phase modulation due to the micro-motions of the target and can be expressed as [15]:

$$
\Phi_{m D}(\eta)=-\frac{2 \pi}{\lambda} \frac{L_{b}}{2} \cos \left(\frac{\beta}{2}\right) \cos (\delta) \cos \left(\boldsymbol{\Omega} \eta+\theta_{0}\right)
$$

where $L_{b}$ is the length of the rotor blade, $\Omega$ is the angular rotation rate and $\theta_{0}$ is the initial rotating phase. The product $\cos \left(\frac{\beta}{2}\right) \cos (\delta)$ is the factor that takes into consideration the bistatic geometry [15].

The amplitude of the received echo is also affected by the rotation of the blade and may be written as:

$$
A_{1}(\eta)=A_{0} \operatorname{sinc}\left(\frac{2 \pi}{\lambda} \frac{L_{b}}{2} \cos \left(\frac{\beta}{2}\right) \cos (\delta) \cos \left(\boldsymbol{\Omega} \eta+\theta_{0}\right)\right)
$$

Assuming that a rotor has $N$ blades they will have $N$ different initial phases $\theta_{k}=\theta_{0}+k 2 \pi / N$ with $k=0,1,2, \ldots, N-1$. 
The total received signal from the $N$ blades will be the sum of the modulated signal from each blade, from (2), (3) and (4) it can be written as:

$$
\begin{aligned}
s_{t o t}(\tau, \eta)= & \sum_{k=0}^{N-1} A_{1_{k}}(\eta) p_{r}\left(\tau-\frac{R(\eta)}{c}\right) \\
& \exp \left\{-j\left(\Phi_{R}(\eta)+\Phi_{m D_{k}}(\eta)\right)\right\}
\end{aligned}
$$

The expression is similar to the signal model in [15] but it is adapted for the specific proposed system. The micro-Doppler shift can be obtained for each blade from (3) as:

$$
\begin{aligned}
& f_{m D_{k}}(\eta)=\frac{1}{4 \pi} \frac{d}{d \eta} \Phi_{m D}(\eta)= \\
& \frac{L_{b}}{2 \lambda} \boldsymbol{\Omega} \cos \left(\frac{\beta}{2}\right) \cos (\delta)\left[-\sin \left(\theta_{k}\right) \sin (\boldsymbol{\Omega} \eta)+\right. \\
& \left.+\cos \left(\theta_{k}\right) \cos (\boldsymbol{\Omega} \eta)\right]
\end{aligned}
$$

Equation (6) represents the expression of the expected micro-Doppler signature for a rotor blade of an helicopter of length $L_{b}$ with a rotation rate $\Omega$, it can be used then to obtain the features of the rotating blade from the micro-Doppler signature.

\section{EXPERIMENTAl Setup AND ACQUisitions}

In this section the experimental setup and the real data acquisitions are desctribed.

In order to acquire the data a 2 channel GPS receiver was designed with on board computing capabilities. As described in [14] a high gain antenna is required in order to be able to achieve acceptable detection ranges. For this reason an active GPS antenna was selected, the selected PCTEL 3978D-DH is shown in Figure 3, this antenna has a gain of $40 \mathrm{~dB}$ and 0.05 $\mathrm{dB}$ of noise figure of the Low Noise Amplifier.

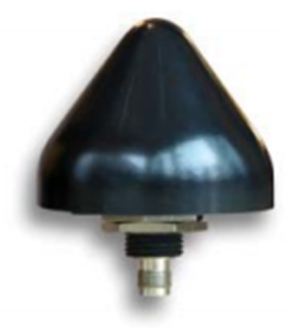

Figure 3: PCTEL 3978D-DH GPS Antenna

The self-built receiver, shown in Figure 4, uses an LNA (Low Noise Amplifier) front end before a downconversion chip containing an LNA, clock generation, mixer and antiimaging filters delivering an IF frequency of $4 \mathrm{MHz}$. The IF frequency is digitised using an 8bit ADC (Analogue to Digital Convertor) using a sampling rate of $16.369 \mathrm{MHz}$. The digitised signal is buffered in a Xilinx Spartan 6 FPGA before it is transferred over USB using a Cypress EZUSB chip. The host software runs on Linux reading data from the USB bus using libusb and writing to a binary file. This binary

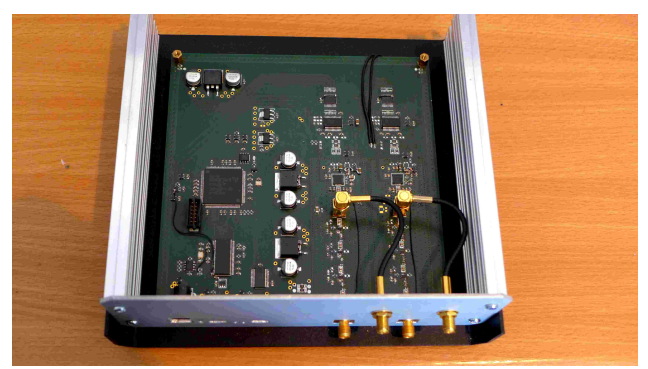

Figure 4: Developed Receiver

file is then easily read and parsed in MATLAB.

The real data acquisitions were performed in proximity of, Cumbernauld Airport in Scotland. The satellites visible at the time of acquisition [25] (with reference to their PRN codes) were: 5, 10, 15, 26 and 28. Measurements were obtained with two different helicopters flying in the area and used for pilot training purposes. For this reason the helicopters were following a set path of taking off performing a circuit of the airport and landing. The two helicopters used were a small two seat Robinson R22 and a larger four seat Robinson R44, both use two bladed main and tail rotors [26]. The R22 has a main rotor repetition of 8.5 RPS (Rotations Per Second), the tail rotor has a repetition rate of 53.3 RPS. The motor rotor blades are $3.84 \mathrm{~m}$ in length and the tail rotor has a length of $0.53 \mathrm{~m}$. The R44 main rotor has a rotation rate of 6.8 RPS with a blade length of $5.03 \mathrm{~m}$. The tail rotor has a repetition rate of 40.5 RPS with a blade length of $0.74 \mathrm{~m}$.

\section{EXPERIMENTAL RESULTS}

In this section we present the results obtained from the real data acquired while the R22 and R44 helicopters were flying approaching and landing to Cumbernauld Airport.

To process the data the GPS satellite showing the best bistatic geometry (i.e. providing forward scattering enhancement) was selected using the real time information on the position of the GPS satellites, at the time of acquisition [25]. Considering the flight path of the helicopters, satellite number 26 was identified as the one providing the best geometry, considering the flight path of the helicopters. Notice that the selection of the best geometry can be performed by the receiver in an operative scenario, for example a specific selection can be used if the interest is to monitor a given area or all the available satellites can be used to cover most of the possible directions.

The received signals are then cross-correlated with the C/A sequence of the selected satellite. In order to remove the direct signal and the other signal components (i.e. fuselage return) the Singular Value Decomposition technique proposed in [23] is used.

In Figure 5 the spectrogram of the extracted micro-Doppler signature of the main rotor of the R44 is shown. By measuring the interval between two flashes it is possible to extract an interval of $0.0735 \mathrm{~s}$ leading to 13.6 flashes per seconds. The signature shows that an even number of blades are present 
in the rotor, thus the 13.6 flashes per second correspond to 6.8 RPS for a 2 bladed helicopter. This value matches the characteristics of the main rotor of the R44 helicopter.

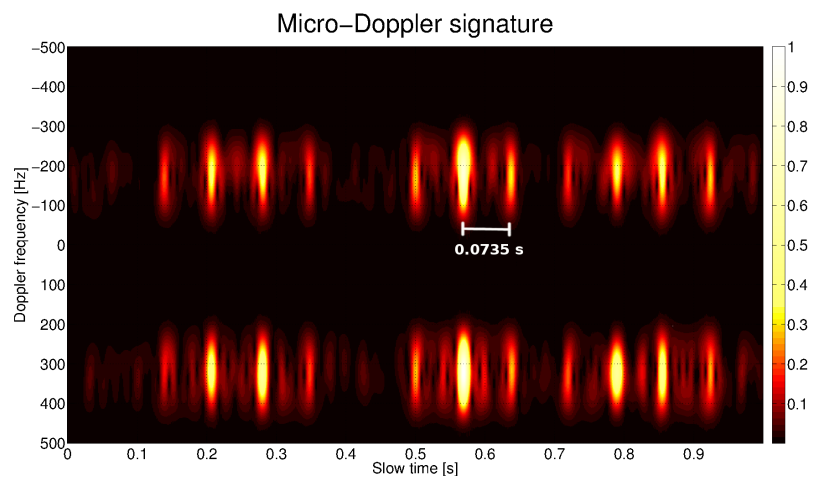

Figure 5: Micro-Doppler Signature of the main rotor of the R44 Helicopter

The same processing has been applied to the returns collected while the R22 was flying. The spectrogram shown in Figure 6, results less clear than the one shown in Figure 5. However from the top of the spectrogram the periodic component due to the flash of the tip of the blade is visible. The separation between two consecutive flashes is $0.0599 \mathrm{~s}$ leading to 16.69 flashes per second. Assuming 2 blades in the rotor this leads to 8.34 RPS in agreement with the possible values of RPS around 8.5 RPS reported in the pilot manual of the R22 helicopter [26].

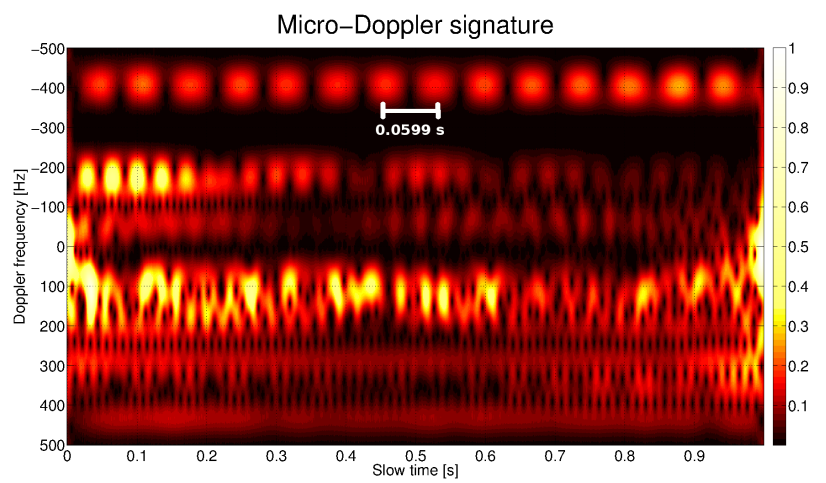

Figure 6: Micro-Doppler Signature of the main rotor of the R22 Helicopter

\section{CONCLUSIONS}

This paper presented the experimental validation of the concept proposed in [14]. The capability of extracting microDoppler signatures of main rotor of helicopters exploiting GNSS signals of opportunity has been demonstrated through the acquisition of real signals from 2 helicopters flying in the Cumbernauld Airport area. The results demonstrate that in the near forward scattering regime the main rotor returns can be detected and that the micro-Doppler signature can be used for helicopters identification. Further development will see the exploitation of the echoes with multiple PRNS at the same time, and the investigation of the best strategy for the signature extraction and classification, including sparse and model based approaches.

\section{ACKNOWLEDGMENT}

This work was supported by the Engineering and Physical Sciences Research Council (EPSRC) Grant number EP/K014307/1 and the MOD University Defence Research Collaboration in Signal Processing.

\section{REFERENCES}

[1] H.D. Griffiths and C.J. Baker, "Passive coherent location radar systems. part 1: performance prediction," Radar, Sonar and Navigation, IEE Proceedings -, vol. 152, no. 3, pp. 153-159, June 2005.

[2] D. W. O'Hagan, A. Capria, D. Petri, V. Kubica, M. Greco, F. Berizzi, and A. G. Stove, "Passive bistatic radar (pbr) for harbour protection applications," in Radar Conference (RADAR), 2012 IEEE, May 2012, pp. 0446-0450.

[3] D.W. O’Hagan, F. Colone, C.J. Baker, and H.D. Griffiths, "Passive bistatic radar (pbr) demonstrator," in Radar Systems, 2007 IET International Conference on, Oct 2007, pp. 1-5.

[4] G.E. Smith, J.L. Garry, R.L. Ewing, and C.J. Baker, "Ambiguity analysis of hd fm waveforms for use in passive radar," Electronics Letters, vol. 49, no. 18, pp. 1178-1179, August 2013.

[5] P. Falcone, F. Colone, C. Bongioanni, and P. Lombardo, "Experimental results for ofdm wifi-based passive bistatic radar," in Radar Conference, 2010 IEEE, May 2010, pp. 516-521.

[6] F. Colone, P. Falcone, C. Bongioanni, and P. Lombardo, "Wifibased passive bistatic radar: Data processing schemes and experimental results," Aerospace and Electronic Systems, IEEE Transactions on, vol. 48, no. 2, pp. 1061-1079, APRIL 2012.

[7] M. Malanowski, K. Kulpa, J. Kulpa, P. Samczynski, and J. Misiurewicz, "Analysis of detection range of fm-based passive radar," Radar, Sonar Navigation, IET, vol. 8, no. 2, pp. 153-159, February 2014.

[8] K. Chetty, G.E. Smith, and K. Woodbridge, "Through-the-wall sensing of personnel using passive bistatic wifi radar at standoff distances," Geoscience and Remote Sensing, IEEE Transactions on, vol. 50, no. 4, pp. 1218-1226, April 2012.

[9] M. Antoniou and M. Cherniakov, "Experimental demonstration of passive gnss-based sar imaging modes," in Radar Conference 2013, IET International, April 2013, pp. 1-5.

[10] D. Olivadese, E. Giusti, D. Petri, M. Martorella, A. Capria, and F. Berizzi, "Passive isar with dvb-t signals," Geoscience and Remote Sensing, IEEE Transactions on, vol. 51, no. 8, pp. 4508-4517, Aug 2013.

[11] Jun Liu, Hongbin Li, and B. Himed, "Two target detection algorithms for passive multistatic radar," IEEE Transactions on Signal Processing, vol. 62, no. 22, pp. 5930-5939, Nov 2014

[12] Guolong Cui, Jun Liu, Hongbin Li, and Braham Himed, "Signal detection with noisy reference for passive sensing," Signal Processing, vol. 108, pp. 389, Ä̂̂399, Mar 2015.

[13] Jonathan Pisane, Sylvain Azarian, Marc Lesturgie, and Jacques Verly, "Automatic target recognition for passive radar," Aerospace and Electronic Systems, IEEE Transactions on, vol. 50, no. 1, pp. 371-392, January 2014.

[14] C. Clemente and John J. S., "GNSS-Based Passive Bistatic Radar for Micro-Doppler Analysis of Helicopter Rotor Blades," IEEE Transactions on Aerospace and Electronic Systems, vol. 50, no. 1, pp. 491-500, January 2014.

[15] V. Chen, Micro-Doppler Effect in Radar, Artech House, first edition, 2011.

[16] C. Clemente and John J. Soraghan, "Vibrating Target Micro-Doppler Signature in Bistatic SAR with a Fixed Receiver," Geoscience and Remote Sensing, IEEE Transactions on, vol. 50, no. 8, pp. 3219-3227, Aug 2012

[17] G.E. Smith, K. Woodbridge, C.J. Baker, and H. Griffiths, "Multistatic micro-doppler radar signatures of personnel targets," Signal Processing, IET, vol. 4, no. 3, pp. $224-233$, june 2010.

[18] C. Clemente, A. Balleri, K. Woodbridge, and J.J. Soraghan, "Developments in target micro-doppler signatures analysis," EURASIP Journal on Advances in Signal Processing, vol. 2013, no. 1, 2013. 
[19] L. Pallotta, C. Clemente, A. De Maio, J. Soraghan, and A. Farina, "Pseudo-Zernike Moments Based Radar Micro-Doppler Classification," in IEEE Radar Conference, Cincinnati, USA, 19-23 May 2014.

[20] B.C. Barber, "Imaging the rotor blades of hovering helicopters with SAR," in Radar Conference, 2008. RADAR '08. IEEE, may 2008, pp. $1-6$.

[21] Anria Cilliers and Willem A J Nei, "Helicopter parameter extraction using joint time-frequency and tomographic techniques," in Proceedings of the International Conference on Radar, 2008. Sept. 2008, pp. 598603, Institute of Electrical and Electronic Engineers.

[22] M.K. Baczyk, J. Misiurewicz, D. Gromek, and K. Kulpa, "Analysis of recorded helicopter echo in a passive bistatic radar," in Radar Conference (EuRAD), 2013 European, Oct 2013, pp. 243-246.

[23] C. Clemente and J. J. Soraghan, "Application of the singular spectrum analysis for extraction of micro-Doppler signature of helicopters," in IET International Conference on Radar Systems (Radar 2012), Oct 2012, pp. $1-5$.

[24] Nicholas J. Willis, Bistatic Radar, Artech House, 1995.

[25] "Map of live satellite positions, in-the-sky.org," http://in-thesky.org/satmap.php.

[26] "Robinson helicopter company," http://www.robinsonheli.com/. 